\title{
Study on the impact of multidimensional proximity on the innovation of tourism industry cluster
}

\author{
Zhang Hua ${ }^{1 *}$, Sun Peng ${ }^{2}$ \\ ${ }^{1}$ Zhang Hua $1 \mathrm{Xi}$ 'an International Studies University, Department of Tourist Management, Xi an, Shaanxi, China \\ ${ }^{2}$ Sun Peng $2 \mathrm{Xi}$ an International Studies University, Department of Tourist Management, Xi`an, Shaanxi, China
}

\begin{abstract}
This article from the perspective of regional economics and human geography, tries to explore the influence mechanism of tourism industry cluster innovation by using the latest research results of "proximity" in the west. Based on the analysis of relevant theories, This paper proposes relevant research hypotheses, which enriches the theories related to proximity and industrial cluster innovation and lays a theoretical foundation for exploring the essential factors affecting the innovation of tourism industry cluster.
\end{abstract}

\section{Introduction}

Since the end of 1990s, the "relationship turn" in economic geography has focused people's attention on the study of cluster innovation from the relationship between inside and outside organizations. Cluster innovation is influenced by many driving factors, and proximity is a new perspective. With the deepening of the research on multidimensional proximity in various fields, areas such as economic geography have developed the concept of proximity to explain the network of industrial areas and the formation of innovative clusters. At present, although the literature on cluster innovation has traditionally focused on manufacturing industrial clusters, this trend has changed and the innovation of tourism clusters has become a fairly important field due to their unique characteristics. Tourism, as a hugely innovative phenomenon, in the dynamic business environment of increasing globalization and fierce competition between domestic and foreign travel destinations, finding new ideas and innovation has become the fundamental for tourism companies to gain competitive advantages. The role of proximity in promoting collaborative innovation among participants has been widely recognized in the tourism literature, Especially through the application of the concepts of industrial zone and cluster ${ }^{[1]}$, but the research on tourism industry based on the proximity theory is almost blank. Therefore, based on the influence of proximity on the innovation of industrial clusters, Combined with the development characteristics of tourism industry clusters, This paper explores the influence of proximity on the innovation of tourism industry clusters and puts forward a hypothesis.

\section{Review of related research}

\subsection{Proximity}

Proximity originated from the concept of cluster economy proposed by Marshall, and it mainly refers to the spatial co-positioning relationship between economic activity subjects in the same cluster, that is, geographical proximity. As the economic globalization, the rapid development of information and communication technology and the booming of knowledge economy, in the 1980s, the "new industrial district", in a single geographic proximity analysis area the limitation of the development issue is more and more prominent, geography is no longer "important", "geography is dead" ${ }^{[6]}$ question appear constantly; In the 1990s, scholars represented by the school of proximity dynamics in France raised objections to the impact of geographic proximity on innovation. They pointed out that there are other dimensions (institution, cognition, technology, society, relationship, etc.) that affect innovation development. Since then, many western scholars have begun to discuss the issue of multidimensional proximity and innovation. Since then, many western scholars have begun to discuss the issue of multidimensional proximity and innovation. The concept of proximity is multifaceted. Bosma divided it into five dimensions: geography, cognition, organization, institution and social proximity. This paper will use Boschma's five dimensions to distinguish proximity.

\footnotetext{
* Corresponding author: 1030236448@qq.com
} 


\subsection{Research status of cluster innovation from the perspective of proximity}

\subsubsection{Research Status of Foreign Countries}

Foreign academic research on industrial clusters based on the perspective of multidimensional proximity started earlier.It mainly includes the following aspects: (1) The construction of the theoretical framework of proximity. Andr Torre Shaw et al. (2000) introduced the research results of the French Proximity Dynamics School, aiming to emphasize some major problems in the definition of the Proximity Theory [2].Boschma(2005) proposed a proximity framework for inter-organizational relationship types that are expected to promote interactive learning and collaborative innovation ${ }^{[3]}$; Pierre-Alexandre Balland (2015) proposed a dynamic perspective based on the co-evolution of knowledge network and proximity to extend the analysis framework of proximity [4];(2) Research on the influence mechanism of multi-dimensional proximity on cluster innovation. As the proposer of Evolutionary Economic Geography and the master of related studies, Boschma (2005) stated that geographical proximity can strengthen the role of other proximity and innovation, and institutional proximity to a large extent affects the internal and external organizational proximity of clusters ${ }^{[3]}$.Giuliani (2007) emphasized the influence of cognitive proximity on knowledge flow ${ }^{[4]}$; Teis Hansen. (2014) discussed the influence of cooperation motivation on the proximity characteristics of partnership ideal in innovation projects [5].Geldes (2017) Research on the determinants of business cooperation between enterprises and other organizations based on proximity method ${ }^{[6]}$;Capone \& Lazzeretti (2018) explored the different roles of proximity in a variety of informal network relationships ${ }^{[7]}$;(3) Analysis of the dynamic impact of multidimensional proximity on cluster innovation. Letaifa \& Rabeau (2013) studied the possible negative effects of different proximity dimensions, and proved that excessive cognitive and institutional proximity would have a negative impact on relationship creation in the advanced stage of cluster life cycle ${ }^{[9]}$.Molina-Morales (2015) et al. used the ERGM model to analyze a food cluster in Spain, aiming to clarify the possible harmful effects and complementarities between adjacent regions ${ }^{[10]}$.

\subsubsection{Domestic Research Status}

The research on industrial cluster innovation based on the perspective of "proximity" started relatively late in China. The specific research content mainly focuses on the following aspects: (1)Basic theoretical research and construction of theoretical framework. In early China, some scholars discussed the relationship between geographical proximity and regional and industrial cluster innovation. Wang Jici(2005) analyzed the proximity and agglomeration of enterprises in the development of industrial clusters and industrial parks ${ }^{[11]}$.Li Lin and Han Baolong (2009) reclassified and defined the different dimensions of multi-dimensional proximity from the perspective of organizational cooperation $^{[12]}$.Wang Tao and Zeng Gang (2008) discussed the effect mechanism of geographical proximity factors on enterprise innovation activities at local and regional levels ${ }^{[13]}$.(2) Empirical analysis of the action mechanism and influence mechanism of multidimensional proximity on industrial cluster innovation. Wang Xiaobin and Li Fugang (2007) discussed the mechanism of geographical proximity in regional innovation from the perspective of knowledge flow and collective learning ${ }^{[14]}$. Li Erling and Li Xiaojian (2016) explore the geographical proximity and relational proximity characteristics of vegetable cluster enterprises and their influences on the innovation of cluster enterprises ${ }^{[15]}$.Zhou Lichun (2018)analyzed the mechanism of organizational proximity on the innovation of advertising industrial clusters from the perspectives of organizational structure, institutional constraints and social relations ${ }^{[16]}$. (3) Analysis of the dynamic impact of multidimensional proximity on cluster innovation. Li Lin and Xiong Xuemei (2012) analyzed the dynamic influence mechanism of geographic proximity on innovation in different stages of cluster evolution from the perspective of industrial cluster life cycle ${ }^{[17]}$.Ye Qin, Zeng Gang(2017) studied the influence of organizational and cognitive proximity on the evolution of innovation network of petroleum equipment manufacturing industry in Dongying City ${ }^{[18]} . \mathrm{Li}$ Lin, Deng $\mathrm{Ru}$ et al. (2018)showed that geographical proximity and cognitive proximity had different impacts on innovation in different stages of cluster evolution ${ }^{[19]}$.

Throughout the research on industrial clusters based on proximity, it can be seen that the domestic and foreign academic circles are experiencing a deepening process from static to dynamic, from theory to demonstration. In terms of research fields, the domestic and foreign related studies are mainly focused on high value-added industries and high-tech industrial clusters.

\subsubsection{Domestic Research Status}

As a service-oriented industry, tourism has a big gap with high-tech industry and high value-added industry in the development process. Therefore, the influencing factors that promote their innovation are also different. Recent studies have pointed to the role of proximity in the development of the tourism industry. Among them, Adi Weidenfeld (2013) points out that the social and institutional proximity is reflected by the differences between formal institutions (laws and regulations) and informal institutions (practices and habits) as well as the historical track. These aspects influence the exchange of tacit knowledge. They also determine the types of tourism developed on both sides of the border and constitute non-geographic dimensions of proximity, including cognitive, organizational, social, institutional, cultural and technological dimensions that affect knowledge transfer between enterprises ${ }^{[20]}$.Ingrid Koflera and Anja Marcher (2018) points out that the promoting effect of proximity on collaborative innovation among 
participants has been widely recognized in the tourism literature, especially through the application of the concepts of industrial zones and clusters ${ }^{[21]}$.

Although with the deepening of tourism research, Many scholars in the academic circle have tried to study the innovation of tourism industry cluster, but the research on the innovation of tourism industry cluster based on the perspective of proximity is still in a blank state.

\section{Influence mechanism analysis of multidimensional proximity on innovation of tourism industry cluster}

Tourism is a complex system, this complexity and dynamism is manifested in the interrelationships and interdependence between travel companies and other industries, such as financial or intermediary services. At the same time, tourism plays a key role in the environment, society and cultural atmosphere of the destination. As a result, Tourism is an activity that sustains multiple interactions, not only in the economic sector, but also in the cultural, environmental and social spheres. With the passage of time, due to the limitation of local tourism resources and the prevalence of tourism activities, the enterprise alliance of tourism destinations is not limited to geographically adjacent enterprises. In order to explore new information and knowledge from different contacts and avoid lock-in issues, it is possible to transcend geospatial limitations and to establish relationships and form ongoing interactions with different agents of non-local enterprises in a wider range of local, national and international contexts. This emphasizes the impact of proximity dimensions -cognition, organization, institution, and society -- on the innovation of tourism industrial clusters in addition to geographical proximity.

\subsection{The Influence of Geographical Proximity on the Innovation of Tourism Industry Cluster}

Geographical proximity is considered to be a key factor in promoting the dissemination of local knowledge. Literature on regional cluster development emphasizes that geographical proximity promotes localized learning as a way to gain competitive advantage. Different from other productive industries, tourism is highly dependent on local resources and has strong regional and historical characteristics. Therefore, region is the main factor in the development of tourism industry, and its main characteristic is to take territory into consideration as a central element. Due to the dependence of tourism enterprises on territory in the process of development, Are not keen to keep in touch with other similar travel companies in terms of innovation needs, more willing to cooperate within its immediate geographical boundaries and thus to be rooted in territory and destinations. Therefore, geographical proximity is one of the important dimensions of its innovation and development. Space becomes an important factor in the analysis of tourism enterprises. With the tourism industry cluster as the core, spatial proximity promotes frequent and close relationships between businesses and allows for "face to face" interaction. In particular, due to the spatial proximity of enterprises in the tourism industry, it is possible to optimize the horizontal relationship of complementarity and competition in the tourism industry, as well as the vertical and diagonal relationships, which makes the development of informal interaction between potential partners more effective. Therefore, this paper proposes Hypothesis 1:

Geographical proximity has a positive impact on the innovative development of tourism industry clusters

\subsection{The Influence of Organizational Proximity on the Innovation of Tourism Industry Cluster}

The essence of organizational proximity is the network connection of various actors in the industrial agglomeration area under the same system, society, culture or organization arrangement. In the process of enterprise development, it tends to establish contact with the same enterprise group or participants who have cooperative experience. The organization content of both partners is similar, and the proximity of the organization can promote the cooperation between enterprises, so that the tacit knowledge and other non-standardized resources of the partners can flow and transfer more smoothly, thus promoting cooperative innovation. Studies have shown that tourism enterprises can take advantage of the diversity of their inter-organizational relationships to acquire relevant exploratory knowledge and thus produce more fundamental innovations. In addition, tourism enterprises interact with different agents and agencies belonging to the tourism destination and participate in the destination community together, that is, these companies should increase the diversity of their relationships mainly by extending their relationships to distant and unconnected enterprises. These diverse inter-organizational relationships can help bring together companies to build Bridges and bonds that are conducive to the acquisition of new and creative knowledge, which is key to developing fundamental innovation, which in turn will help them compete in the global environment of tourism. Therefore, this paper proposes Hypothesis 2:

Organizational proximity has a positive impact on the innovative development of tourism industry clusters

\subsection{The Influence of Cognitive Proximity on the Innovation of Tourism Industry Cluster}

The cognitive dimension consists of shared values and goals. The potential benefits of shared goals and values can be reinforced by the perception of dramatic market changes in a clustered business. As we all know, a common culture is essential to enhance the exchange and understanding of complex market information. Therefore, a shared culture promotes collective work because it helps to understand the thoughts and thoughts expressed by agents in the peer group. In the process of development of various subjects within an industrial 
cluster, the similarity of knowledge base among partners increases mutual understanding and communication, promotes the flow and transfer of knowledge, and reduces transaction costs ${ }^{[11]}$, thus optimizing the degree of mutual learning among partners. At the same time, relying on relatives, friends, teachers and students (kinship, industry, geographical advantage) can also promote the development of cognitive proximity. Tourism cluster enterprises may be influenced by managers' perception of market vitality in the process of innovation. This is because, for tourism enterprises, entrepreneurship has been considered as the key to innovation activities of tourism enterprises. As Schumpeter (1934) argued, entrepreneurs create a constant disturbance to the market equilibrium. Entrepreneurs are "creative destroyers" who set new standards with their ideas, products, and ideas, and fundamentally alter customer tastes and preferences with their innovation. Entrepreneurship is the key factor to transform tourism products to the direction of evolution and improve competitiveness. In addition, in the tourism industry, shared technical capabilities and similar educational backgrounds can promote knowledge transfer between different economic sectors, thus realizing the recombination of existing technologies. At the same time, at the regional level, the sharing of natural and cultural resources between neighboring enterprises increases the shared knowledge base among participants, thus promoting the development of common tourism. Therefore, this paper proposes Hypothesis 3:

Cognitive proximity has a positive impact on the innovative development of tourism industry clusters

\subsection{The Influence of Institutional Proximity on the Innovation of Tourism Industry Cluster}

Institutional proximity is widely regarded as a key component of innovation systems. Both formal institutions (laws and rules) and informal institutions (cultural norms and values) play an important role in determining how participants relate to each other and how they learn and use knowledge. In personal life and in the work of companies and other organizations, Institutions that enhance order, trust and predictability are important to competitiveness and innovation. Institutional proximity emphasizes that the economic behavior of firms in clusters is influenced by the similarity of formal and informal institutions. As far as tourism is concerned, on the one hand, the formal system mainly refers to the influence of the similarity of policies, regulations and management measures within the cluster on the innovation cooperation of tourism enterprises. On the other hand, informal system mainly refers to the influence of the same social culture and norms, industrial atmosphere, and business practices in the cluster on the innovation cooperation of tourism enterprises. In the process of industrial cluster development, the positive effect of institutional proximity can be used to "glue" the collective actions of individuals or organizations together, which can reduce uncertainty, communication costs and transaction costs, and thus promote the transfer of knowledge, especially tacit knowledge. This kind of institutional proximity includes not only the institutional proximity between the main enterprise and the cooperative enterprise and the scientific research institution, but also the institutional proximity between the government and so on. Therefore, in the process of industrial cluster development, Institutions that enhance order, trust and predictability are important to competitiveness and innovation. Therefore, this paper proposes hypothesis 4:

Institutional proximity has a positive impact on the innovative development of tourism industry clusters

\subsection{The Influence of Social Proximity on the Innovation of Tourism Industry Cluster}

Social proximity explains most of the spillover effects. It is based on the observation that economic relationships may reflect social relationships (friendships, kinship, shared past experiences, etc.) on a micro level, and has to do with trust-based interactions between business actors--trust promotes cooperation and tacit knowledge exchange, making knowledge transfer more effective. This climate of trust arises from inherent geographical proximity--face-to-face interaction $s$ that lead to knowledge sharing and cooperative behavior; Social proximity is usually associated with interpersonal relationships between participants ${ }^{[33]}$ (for example, arising from friendship or family ties) and mainly reflects the strength of social ties; Social proximity can also be revealed by past cooperation, for example, by observing repeated relationships or whether two potential partners had a common third party partner in the past.

Social proximity is mainly reflected through social network. Social network is the inter-organizational relationship established between travel companies and other travel agents, which is regarded as the key determinant of travel companies' innovation. Recently, due to the importance of social networks to enterprise innovation, and therefore to destination competitiveness, the social networking approach has been applied at the enterprise level of the tourism industry ${ }^{[3]}$.Social networks are seen as crucial in mobilizing information and resources, as well as cooperation between tourism businesses. In this context, the network organization and collaboration of the tourism sector is extremely important, as these networks facilitate the dissemination of information, strengthen the links between individuals and organizations, contribute to more sustainable tourism development, greater tourist satisfaction and the economic success of the areas in which they operate. Therefore, this paper proposes Hypothesis 5:

Social proximity has a positive impact on the innovative development of tourism industry clusters

\section{Conclusion}

This paper, based on the perspective of multidimensional proximity, combined with the characteristics of 
tourism industry cluster, preliminary discussion on the different dimensions of proximity effect on tourism industrial cluster innovation and development, and proposed the corresponding hypothesis theory, for the multidimensional proximity and industrial cluster innovation research provides a new perspective, to study factors influencing the nature of tourism industrial cluster innovation provides the valid path, has a certain practical significance. In the future, field research will be carried out in combination with tourism industry cluster enterprises, and empirical analysis will be conducted with appropriate research methods to verify the above research hypotheses.

\section{Acknowledgments}

This research was supported by 2019 Federation of Socia 1 Science Foundation of Shaanxi Province (2019C129), 2 020 Social Science Planning Foundation of Xi\&apos;an (JG90), 2021 Shaanxi Province Soft Science Research Pr oject(2021KRM152) and Postgraduate Foundation of Xi \&apos;an International Studies University (SYJS201828 , SSZD2019020)

\section{References}

1. Zach, F.J., \& Hill, T. L. (2017). Network, knowledge and relationship impacts on in novation in tourism destinations. Tourism Management, 62, 196-207

2. Torre A, Gilly J P. On the analytical dimension of proximity dynamics [J]. Regional Studies, 2000, 34(2): $169-180$

3. BOSCHMA R: Proximity and innovation: a critical assessment[J]. Regional Studies, 2005, 39: 61-74.

4. Pierre-Alexandre Balland, Ron Boschma, Koen Frenken. Proximity and Innovation: From Statics to Dynamics. 2015, 49(6): 907-920.

5. Giuliani, E. 2007. "Networks and Heterogeneous Performance of Cluster Firms." In Applied Evolutionary Economics and Economic Geography, edited by Koen Frenken, 161-179.

6. Teis Hansen. Juggling with Proximity and Distance: Collaborative Innovation Projects in the D anish Cleantech Industry. 2014, 90(4):375-402.

7. Cristian Geldes, Jorge Heredia, Christian Felzensztein, Marcos Mora, (2017) "Proximity as determinant of business cooperation for technological and non-technological innovations: a study of an agribusiness cluster", Journal of Business \& Industrial Marketing, Vol. 32 Issue: 1, pp.167-17

8. Molina-Morales, F. X., Belso-Martinez, J. A., MasVerdù, F., \& Martinez-Chàfer, L. (2015). Formation and dissolution on inter-firm linkages in lengthy and stable networks in clusters. Journal of Business Research, 68, 1557 - 1562.

9. Ben Letaifa, S., and Y. Rabeau. 2013. “Too close to Collaborate? How Geographic Proximity Could
Impede Entrepreneurship and Innovation.” Journal of Business Research 66: 2071 - 2078.

10. Molina-Morales, F. X., Belso-Martinez, J. A., MasVerdù, F., \& Martinez-Chàfer, L. (2015). Formation and dissolution on inter-firm linkages in lengthy and stable networks in clusters. Journal of Business Research, 68, 1557 - 1562.

11. Wang Jici.Analysis on the proximity and agglomeration of enterprises in the development of industrial clusters and industrial parks [J]. China Soft Science,2005(12):91-98.

12. Li Lin, Han Baolong. Multi-dimensional proximity in organizational cooperation: A review and thinking of western literature [J].Social Scientist, 2009(07):108-112.

13. Wang Tao, Zeng Gang.Research on the innovation activities of high-tech enterprises in Shanghai Pudong: A case study of Lower Saxony [J].World Regional Studies,2008(01):47-52+84.

14. Wang Xiaobin, Li Fugang. The role of geographic proximity in regional innovation and its implications [J].Economic Geography,2007(04): 543 -546+552.

15. Yanwen Shi, Erling Li, Xiaojian Li.The impact of geographical proximity and relational proximity on the innovation of agricultural industrial clusters: A case study of Shouguang vegetable industry cluster in Shandong Province [J].Scientia Geographica Sinica, 2016,36(5):751-759

16. Lichun Zhou.The influence of organizational proximity on the innovation of advertising industry clusters: A case study of national advertising industrial parks [J].Journal of Communication University of China,2018,40(10):7-13.

17. Li Lin, Xiong Xuemei.The dynamic impact of geographic proximity on cluster innovation from the perspective of industrial cluster life cycle: A case study of China's automobile industry cluster [J].Geographical Research,2012,31(11):2017-2030.

18. Chen Hongting, Ye Qin, Zeng Gang. The influence of organizational proximity on innovation network evolution of petroleum equipment manufacturing industry in Dongying city [J].Human Geography,2017,32(01):116-122.

19. Li Lin, Deng Ru.The dynamic impact of multidimensional proximity on cluster innovation from the perspective of industry life cycle: A case study of China's electronic information industry clusters [J].Soft science, 2018, 32 (08) : 24-27 + 62.

20. Francesco Capone \& Luciana Lazzeretti (2018) The different roles of proximity in multiple informal network relationships: evidence from the cluster of high technology applied to cultural goods in Tuscany, Industry and Innovation, 25:9, 897-917.

21. Belso-Martínez, J. A., \& Molina-Morales, F. X. (2011). The drivers of the open district development: A social capital approach. Regional Science Policy \& Practice, 3(2), 49 - 70 